Bull. Austral. Math. Soc.

$26 \mathrm{D} 15,41 \mathrm{~A} 55$

VoL. 60 (1999) [495-508]

\title{
THE OSTROWSKI INTEGRAL INEQUALITY FOR MAPPINGS OF BOUNDED VARIATION
}

\section{S.S. DRAGOMIR}

A generalisation of the Ostrowski integral inequality for mappings of bounded variation and applications for general quadrature formulae are given.

\section{INTRODUCTION}

In 1938, Ostrowski proved the following integral inequality [7, p.468]

THEOREM 1. Let $f:[a, b] \rightarrow \mathbf{R}$ be continuous on $[a, b]$ and differentiable on $(a, b)$ with derivative $f^{\prime}:(a, b) \rightarrow \mathbf{R}$ is bounded on $(a, b)$, that is, $\left\|f^{\prime}\right\|_{\infty}:=\sup _{t \in(a, b)}\left|f^{\prime}(t)\right|<\infty$. Then the inequality

$$
\left|f(x)-\frac{1}{b-a} \int_{a}^{b} f(t) d t\right| \leqslant\left[\frac{1}{4}+\frac{\left(x-\frac{a+b}{2}\right)^{2}}{(b-a)^{2}}\right](b-a)\left\|f^{\prime}\right\|_{\infty}
$$

hold for all $x \in[a, b]$. The constant $1 / 4$ is the best possible.

For some generalisations and related results see the book [7, p.468-484].

In [3], Dragomir and Wang obtained the inequality

$$
\left|f(x)-\frac{1}{b-a} \int_{a}^{b} f(t) d t\right| \leqslant\left[\frac{1}{2}+\frac{\left|x-\frac{a+b}{2}\right|}{b-a}\right]\left\|f^{\prime}\right\|_{1}
$$

for all $x \in[a, b]$, provided $f$ is continuous on $[a, b]$ and differentiable on $(a, b)$ with derivative $f^{\prime} \in L_{1}(a, b)$.

Note that this result can also be obtained from Fink's theorem [7, Theorem 1, p.471] for $n=1$ and appropriate computations.

Some applications of the above results in numerical integration and for special means have been given in $[3,4,5,6]$.

In the recent paper [7], Dragomir pointed out the following natural generalisation of the above result for the class of mappings of bounded variation.

Received 23rd March, 1999

Copyright Clearance Centre, Inc. Serial-fee code: 0004-9727/99 \$A2.00+0.00. 
THEOREM 2. Let $f:[a, b] \rightarrow \mathbf{R}$ be a mapping of bounded variation on $[a, b]$ and $V_{a}^{b}(f)$ its total variation on $[a, b]$. Then the inequality

$$
\left|f(x)-\frac{1}{b-a} \int_{a}^{b} f(t) d t\right| \leqslant\left[\frac{1}{2}+\frac{\left|x-\frac{a+b}{2}\right|}{b-a}\right] V_{a}^{b}(f)
$$

holds for all $x \in[a, b]$. The constant $1 / 2$ is the best possible.

In this paper we point out a new generalisation of Ostrowski's inequality for mappings of bounded variation and apply it for quadrature formulae in numerical analysis. Some connections with the rectangle, the midpoint and Simpson's rule are also established.

\section{SOME INTEGRAL INEQUALITIES}

We start with the following theorem.

THEOREM 3. Let $I_{k}: a=x_{0}<x_{1}<\ldots<x_{k-1}<x_{k}=b$ be a division of the interval $[a, b]$ and $\alpha_{i}(i=0, \ldots, k+1)$ be $k+2$ points so that $\alpha_{0}=a, \alpha_{i} \in\left[x_{i-1}, x_{i}\right]$ $(i=1, \ldots, k)$ and $\alpha_{k+1}=b$. If $f:[a, b] \rightarrow \mathbf{R}$ is of bounded variation on $[a, b]$, then we have the inequality:

$$
\begin{aligned}
& \left|\int_{a}^{b} f(x) d x-\sum_{i=0}^{k}\left(\alpha_{i+1}-\alpha_{i}\right) f\left(x_{i}\right)\right| \\
& \quad \leqslant\left[\frac{1}{2} \nu(h)+\max \left\{\left|\alpha_{i+1}-\frac{x_{i}+x_{i+1}}{2}\right|, i=0, \ldots, k-1\right\}\right] V_{a}^{b}(f) \\
& \quad \leqslant \nu(h) V_{a}^{b}(f)
\end{aligned}
$$

where $\nu(h):=\max \left\{h_{i} \mid i=0, \ldots, k-1\right\}, h_{i}:=x_{i+1}-x_{i}(i=0, \ldots, k-1)$ and $V_{a}^{b}(f)$ is the total variation of $f$ on the interval $[a, b]$.

Proof: Define the mapping $K:[a, b] \rightarrow \mathbf{R}$ by (see also [1])

$$
K(t):=\left\{\begin{array}{cl}
t-\alpha_{1}, & t \in\left[a, x_{1}\right) \\
t-\alpha_{2}, & t \in\left[x_{1}, x_{2}\right) \\
\vdots & \\
t-\alpha_{k-1}, & t \in\left[x_{k-2}, x_{k-1}\right) \\
t-\alpha_{k}, & t \in\left[x_{k-1}, b\right] .
\end{array}\right.
$$


Integrating by parts using the Riemann-Stieltjes integral, we have successively

$$
\begin{aligned}
\int_{a}^{b} K(t) d f(t)= & \sum_{i=0}^{k-1} \int_{x_{i}}^{x_{i+1}} K(t) d f(t)=\sum_{i=0}^{k-1} \int_{x_{i}}^{x_{i+1}}\left(t-\alpha_{i+1}\right) d f(t) \\
= & \sum_{i=0}^{k-1}\left[\left.\left(t-\alpha_{i+1}\right) f(t)\right|_{x_{i}} ^{x_{i+1}}-\int_{x_{i}}^{x_{i+1}} f(t) d t\right] \\
= & \sum_{i=0}^{k-1}\left[\left(\alpha_{i+1}-x_{i}\right) f\left(x_{i}\right)+\left(x_{i+1}-\alpha_{i+1}\right) f\left(x_{i+1}\right)\right]-\int_{a}^{b} f(t) d t \\
= & \left(\alpha_{1}-a\right) f(a)+\sum_{i=1}^{k-1}\left(\alpha_{i+1}-x_{i}\right) f\left(x_{i}\right)+\sum_{i=0}^{k-2}\left(x_{i+1}-\alpha_{i+1}\right) f\left(x_{i+1}\right) \\
& +\left(b-\alpha_{n}\right) f(b)-\int_{a}^{b} f(t) d t \\
= & \left(\alpha_{1}-a\right) f(a)+\sum_{i=1}^{k-1}\left(\alpha_{i+1}-x_{i}\right) f\left(x_{i}\right)+\sum_{i=1}^{k-1}\left(x_{i}-\alpha_{i}\right) f\left(x_{i}\right) \\
& +\left(b-\alpha_{n}\right) f(b)-\int_{a}^{b} f(t) d t \\
= & \left(\alpha_{1}-a\right) f(a)+\sum_{i=1}^{k-1}\left(\alpha_{i+1}-\alpha_{i}\right) f\left(x_{i}\right)+\left(b-\alpha_{n}\right) f(b)-\int_{a}^{b} f(t) d t \\
= & \sum_{i=0}^{k}\left(\alpha_{i+1}-\alpha_{i}\right) f\left(x_{i+1}\right)-\int_{a}^{b} f(t) d t
\end{aligned}
$$

and then we have the integral equality which is of interest in itself too:

$$
\int_{a}^{b} f(t) d t=\sum_{i=0}^{k}\left(\alpha_{i+1}-\alpha_{i}\right) f\left(x_{i}\right)-\int_{a}^{b} K(t) d f(t) .
$$

On the other hand, we have

$$
\left|\int_{a}^{b} K(t) d f(t)\right|=\left|\sum_{i=0}^{k-1} \int_{x_{i}}^{x_{i+1}} K(t) d f(t)\right| \leqslant \sum_{i=0}^{k-1}\left|\int_{x_{i}}^{x_{i+1}} K(t) d f(t)\right|:=T .
$$


But

$$
\begin{aligned}
\left|\int_{x_{i}}^{x_{i+1}}\left(t-\alpha_{i+1}\right) f(t) d t\right| & \leqslant \sup _{t \in\left[x_{i}, x_{i+1}\right]}\left|t-\alpha_{i+1}\right| V_{x_{i}}^{x_{i+1}}(f) \\
& =\max \left\{\alpha_{i+1}-x_{i}, x_{i+1}-\alpha_{i+1}\right\} V_{x_{i}}^{x_{i+1}}(f) \\
& =\left[\frac{1}{2}\left(x_{i+1}-x_{i}\right)+\left|\alpha_{i+1}-\frac{x_{i}+x_{i+1}}{2}\right|\right] V_{x_{i}}^{x_{i+1}}(f) d t .
\end{aligned}
$$

Then

$$
\begin{aligned}
T & \leqslant \sum_{i=0}^{k-1}\left[\frac{1}{2} h_{i}+\left|\alpha_{i+1}-\frac{x_{i}+x_{i+1}}{2}\right|\right] V_{x_{i}}^{x_{i+1}}(f) \\
& \leqslant \max _{i=0, \ldots, k-1}\left[\frac{1}{2} h_{i}+\left|\alpha_{i+1}-\frac{x_{i}+x_{i+1}}{2}\right|\right] \sum_{i=0}^{k-1} V_{x_{i}}^{x_{i+1}}(f) \\
& \leqslant\left[\frac{1}{2} \nu(h)+\max \left\{\left|\alpha_{i+1}-\frac{x_{i}+x_{i+1}}{2}\right|, i=0, \ldots, k-1\right\}\right] V_{a}^{b}(f)=: V .
\end{aligned}
$$

Now, as

$$
\left|\alpha_{i+1}-\frac{x_{i}+x_{i+1}}{2}\right| \leqslant \frac{1}{2} h_{i}
$$

then

$$
\max \left\{\left|\alpha_{i+1}-\frac{x_{i}+x_{i+1}}{2}\right|, i=0, \ldots, k-1\right\} \leqslant \frac{1}{2} \nu(h)
$$

and consequently,

$$
V \leqslant \nu(h) V_{a}^{b}(f) .
$$

The theorem is completely proved.

Now, if we assume that the points of the division $I_{k}$ are given, then the best inequality we can obtain from Theorem 3 is embodied in the following corollary:

Corollary 1. Let $f$ and $I_{k}$ be as above. Then we have the inequality:

$$
\begin{array}{r}
\mid \int_{a}^{b} f(x) d x-\frac{1}{2}\left[\left(x_{1}-a\right) f(a)+\sum_{i=1}^{k-1}\left(x_{i+1}-x_{i-1}\right) f\left(x_{i}\right)+\left(b-x_{k-1}\right) f(b)\right] \\
\leqslant \frac{1}{2} \nu(h) V_{a}^{b}(f) .
\end{array}
$$

Proof: We choose in Theorem 3,

$$
\begin{aligned}
\alpha_{0} & =a, \alpha_{1}=\frac{a+x_{1}}{2}, \alpha_{2}=\frac{x_{1}+x_{2}}{2}, \ldots, \\
\alpha_{k-1} & =\frac{x_{k-2}+x_{k-1}}{2}, \alpha_{k}=\frac{x_{k-1}+x_{k}}{2} \text { and } \alpha_{k+1}=b .
\end{aligned}
$$


In this case we get

$$
\begin{aligned}
\sum_{i=0}^{k}\left(\alpha_{i+1}-\alpha_{i}\right) f\left(x_{i}\right) \\
=\left(\alpha_{1}-\alpha_{0}\right) f(a)+\left(\alpha_{2}-\alpha_{1}\right) f\left(x_{1}\right)+\ldots+\left(\alpha_{k}-\alpha_{k-1}\right) f\left(x_{k-1}\right)+\left(b-\alpha_{k}\right) f(b) \\
=\quad\left(\frac{a+x_{1}}{2}-a\right) f(a)+\left(\frac{x_{1}+x_{2}}{2}-\frac{a+x_{1}}{2}\right) f\left(x_{1}\right) \\
\quad+\ldots+\left(\frac{x_{k-1}+b}{2}-\frac{x_{k-2}+x_{k-1}}{2}\right) f\left(x_{k-1}\right)+\left(b-\frac{x_{k-1}+b}{2}\right) f(b) \\
=\frac{1}{2}\left[\left(x_{1}-a\right) f(a)+\sum_{i=1}^{k-1}\left(x_{i+1}-x_{i-1}\right) f\left(x_{i}\right)+\left(b-x_{k-1}\right) f(b)\right] .
\end{aligned}
$$

Now, applying the inequality (2.1), we get (2.3).

The following corollary for equidistant partitioning also holds.

Corollary 2. Let

$$
I_{k}: x_{i}:=a+(b-a) \frac{i}{k}(i=0, \ldots, k)
$$

be an equidistant partitioning of $[a, b]$. If $f$ is as above, then we have the inequality:

$$
\begin{array}{r}
\left|\int_{a}^{b} f(x) d x-\left[\frac{1}{k} \cdot \frac{f(a)+f(b)}{2}(b-a)+\frac{(b-a)}{k} \sum_{i=1}^{k-1} f\left[\frac{(k-i) a+i b}{k}\right]\right]\right| \\
\leqslant \frac{1}{2 k}(b-a) V_{a}^{b}(f) .
\end{array}
$$

3. The Convergence of a General Quadrature Formula

Let $\Delta_{n}: a=x_{0}^{(n)}<x_{1}^{(n)}<\ldots<x_{n-1}^{(n)}<x_{n}^{(n)}=b$ be a sequence of division of $[a, b]$ and consider the sequence of numerical integration formulae

$$
I_{n}\left(f, \Delta_{n}, w_{n}\right):=\sum_{j=0}^{n} w_{j}^{(n)} f\left(x_{j}^{(n)}\right)
$$

where $w_{j}^{(n)}(j=0, \ldots, n)$ are the quadrature weights.

The following theorem provides a sufficient condition for the weights $w_{j}^{(n)}$ so that $I_{n}\left(f, \Delta_{n}, w_{n}\right)$ approximates the integral $\int_{a}^{b} f(x) d x$.

THEOREM 4. Let $f:[a, b] \rightarrow \mathbf{R}$ be a mapping of bounded variation on $[a, b]$. If the quadrature weights $w_{j}^{(n)}$ satisfy the condition

$$
x_{i}^{(n)}-a \leqslant \sum_{j=0}^{i} w_{j}^{(n)} \leqslant x_{i+1}^{(n)}-a \text { for all } i=0, \ldots, n-1,
$$


then we have the estimate

$$
\begin{aligned}
& \left|I_{n}\left(f, \Delta_{n}, w_{n}\right)-\int_{a}^{b} f(x) d x\right| \\
& \quad \leqslant\left[\frac{1}{2} \nu\left(h^{(n)}\right)+\max \left\{\left|a+\sum_{j=0}^{i} w_{j}^{(n)}-\frac{x_{i}^{(n)}+x_{i+1}^{(n)}}{2}\right|, i=0, \ldots, n-1\right\}\right] V_{a}^{b}(f) \\
& \quad \leqslant \nu\left(h^{(n)}\right) V_{a}^{b}(f)
\end{aligned}
$$

where $\nu\left(h^{(n)}\right):=\max \left\{h_{i}^{(n)} \mid i=0, \ldots, n-1\right\}$ and $h_{i}^{(n)}:=x_{i+1}^{(n)}-x_{i}^{(n)}$. In particular

$$
\lim _{\nu\left(h^{(n)}\right) \rightarrow 0} I_{n}\left(f, \Delta_{n}, w_{n}\right)=\int_{a}^{b} f(x) d x
$$

uniformly related to the $w_{n}$.

Proof: Define the sequence of real numbers

$$
\alpha_{i+1}^{(n)}:=a+\sum_{j=0}^{i} w_{j}^{(n)}, i=0, \ldots, n
$$

Note that

$$
\alpha_{n+1}^{(n)}=a+\sum_{j=0}^{n} w_{j}^{(n)}=a+b-a=b,
$$

and observe also that $\alpha_{i+1}^{(n)} \in\left[x_{i}^{(n)}, x_{i+1}^{(n)}\right]$.

Define $\alpha_{0}^{(n)}:=a$ and compute

$$
\begin{aligned}
& \alpha_{1}^{(n)}-\alpha_{0}^{(n)}=a, \\
& \alpha_{i+1}^{(n)}-\alpha_{i}^{(n)}=a+\sum_{j=0}^{i} w_{j}^{(n)}-a-\sum_{j=0}^{i-1} w_{j}^{(n)}=w_{i}^{(n)}(i=1, \ldots, n-1), \\
& \alpha_{n+1}^{(n)}-\alpha_{n}^{(n)}=b-\left(a+\sum_{j=0}^{n-1} w_{j}^{(n)}\right)=w_{n}^{(n)} .
\end{aligned}
$$

Then

$$
\sum_{i=0}^{n}\left(\alpha_{i+1}^{(n)}-\alpha_{i}^{(n)}\right) f\left(x_{i}^{(n)}\right)=\sum_{i=0}^{n} w_{i}^{(n)} f\left(x_{i}^{(n)}\right)=I_{n}\left(f, \Delta_{n}, w_{n}\right)
$$

Applying the inequality (2.1), we get the estimate (3.2). 
The uniform convergence related to the quadrature weights $w_{j}^{(n)}$ is obvious by the last inequality.

Now, consider the equidistant partitioning of $[a, b]$ given by

$$
E_{n}: x_{i}^{(n)}:=a+\frac{i}{n}(b-a) \quad(i=0, \ldots, n)
$$

and define the sequence of numerical quadrature formulae

$$
I_{n}\left(f, w_{n}\right):=\sum_{i=0}^{n} w_{i}^{(n)} f\left[a+\frac{i}{n}(b-a)\right] .
$$

The following corollary which can be more useful in practice holds:

COROLlaRY 3. Let $f$ be as above. If the quadrature weight $w_{j}^{(n)}$ satisfy the condition:

$$
\frac{i}{n} \leqslant \frac{1}{b-a} \sum_{j=0}^{i} w_{j}^{(n)} \leqslant \frac{i+1}{n}, i=0, \ldots, n-1
$$

then we have:

$$
\begin{aligned}
& \left|I_{n}\left(f, w_{n}\right)-\int_{a}^{b} f(x) d x\right| \\
& \quad \leqslant\left[\frac{b-a}{2 n}+\max \left\{\left|a+\sum_{j=0}^{i} w_{j}^{(n)}-\frac{2 i+1}{2} \cdot \frac{(b-a)}{n}\right|, i=0, \ldots, n-1\right\}\right] V_{a}^{b}(f) \\
& \quad \leqslant \frac{(b-a)}{n} V_{a}^{b}(f) .
\end{aligned}
$$

In particular, we have the limit

$$
\lim _{n \rightarrow \infty} I_{n}\left(f, w_{n}\right)=\int_{a}^{b} f(x) d x
$$

uniformly related to the $w_{n}$.

\section{Some Particular Integral Inequalities}

The following proposition holds.

PROPOSITION 1. Let $f:[a, b] \rightarrow \mathbf{R}$ be a mapping of bounded variation on $[a, b]$. Then we have the inequality:

$$
\left|\int_{a}^{b} f(x) d x-[(\alpha-a) f(a)+(b-\alpha) f(b)]\right| \leqslant\left[\frac{1}{2}(b-a)+\left|\alpha-\frac{a+b}{2}\right|\right] V_{a}^{b}(f)
$$

for all $\alpha \in[a, b]$. 
The proof follows by Theorem 3 , on choosing $x_{0}=a, x_{1}=b, \alpha_{0}=a, \alpha_{1}=\alpha \in[a, b]$ and $\alpha_{2}=b$.

REMARK 1.

(a) If in (4.1) we put $\alpha=b$, then we get the "left rectangle inequality"

$$
\left|\int_{a}^{b} f(x) d x-(b-a) f(a)\right| \leqslant(b-a) V_{a}^{b}(f)
$$

(b) If $\alpha=a$, then by (4.1) we get the "right rectangle inequality"

$$
\left|\int_{a}^{b} f(x) d x-(b-a) f(b)\right| \leqslant(b-a) V_{a}^{b}(f) ;
$$

(c) It is easly to see that the best inequality we can get from (4.1) is for $\alpha=(a+b) / 2$ obtaining the "trapezoid inequality"

$$
\left|\int_{a}^{b} f(x) d x-\frac{f(a)+f(b)}{2}(b-a)\right| \leqslant \frac{1}{2}(b-a) V_{a}^{b}(f) .
$$

Another proposition with many interesting particular cases is the following one:

PRoposition 2. Let $f$ be as above and $a \leqslant x_{1} \leqslant b, a \leqslant \alpha_{1} \leqslant x_{1} \leqslant \alpha_{2} \leqslant b$. Then we have

$$
\begin{aligned}
& \mid \int_{a}^{b} f(x) d x-\left[\left(\alpha_{1}-a\right) f(a)+\left(\alpha_{2}-\alpha_{1}\right) f\left(x_{1}\right)+\left(b-\alpha_{2}\right) f(b)\right] \\
& \leqslant \frac{1}{2}\left[\frac{1}{2}(b-a)+\left|x_{1}-\frac{a+b}{2}\right|+\left|\alpha_{1}-\frac{a+x_{1}}{2}\right|\right. \\
&\left.+\left|\alpha_{2}-\frac{x_{1}+b}{2}\right|+|| \alpha_{1}-\frac{a+x_{1}}{2}|-| \alpha_{2}-\frac{x_{1}+b}{2}||\right] V_{a}^{b}(f) \\
& \leqslant {\left[\frac{(b-a)}{2}+\left|x_{1}-\frac{a+b}{2}\right|\right] V_{a}^{b}(f) \leqslant(b-a) V_{a}^{b}(f) . }
\end{aligned}
$$

PROOF: Consider the division $a=x_{0} \leqslant x_{1} \leqslant x_{2} \leqslant b$ and the numbers $\alpha_{0}=a, \alpha_{1} \in$ $\left[a, x_{1}\right], \alpha_{2} \in\left[x_{1}, b\right]$ and $\alpha_{3}=b$. Now, applying Theorem 3 , we get

$$
\begin{aligned}
& \left|\int_{a}^{b} f(x) d x-\left[\left(\alpha_{1}-a\right) f(a)+\left(\alpha_{2}-\alpha_{1}\right) f\left(x_{1}\right)+\left(b-\alpha_{2}\right) f(b)\right]\right| \\
& \quad \leqslant \frac{1}{2}\left[\max \left\{x_{1}-a, b-x_{1}\right\}+\max \left\{\left|\alpha_{1}-\frac{a+x_{1}}{2}\right|,\left|\alpha_{2}-\frac{x_{1}+b}{2}\right|\right\}\right] V_{a}^{b}(f)
\end{aligned}
$$




$$
\begin{aligned}
= & {\left[\frac{1}{4}(b-a)+\frac{1}{2}\left|x_{1}-\frac{a+b}{2}\right|+\frac{1}{2}\left|\alpha_{1}-\frac{a+x_{1}}{2}\right|\right.} \\
& \left.+\frac{1}{2}\left|\alpha_{2}-\frac{x_{1}+b}{2}\right|+\frac{1}{2}|| \alpha_{1}-\frac{a+x_{1}}{2}|-| \alpha_{2}-\frac{x_{1}+b}{2}||\right] V_{\mathrm{a}}^{b}(f)
\end{aligned}
$$

and the first inequality in (4.5) is proved.

Now, observe that

$$
\left|\alpha_{1}-\frac{a+x_{1}}{2}\right| \leqslant \frac{x_{1}-a}{2},\left|\alpha_{2}-\frac{x_{1}+b}{2}\right| \leqslant \frac{b-x_{1}}{2} .
$$

Consequently,

$$
\max \left\{\left|\alpha_{1}-\frac{a+x_{1}}{2}\right|,\left|\alpha_{2}-\frac{x_{1}+b}{2}\right|\right\} \leqslant \frac{1}{2} \max \left\{x_{1}-a, b-x_{1}\right\}
$$

and the second inequality in (4.5) is proved.

The last inequality is obvious.

REMARK 2. If we choose above $\alpha_{1}=a, \alpha_{2}=b$, then we get the following Ostrowski type inequality obtained by Dragomir in the recent paper [2]:

$$
\left|\int_{a}^{b} f(x) d x-(b-a) f\left(x_{1}\right)\right| \leqslant\left[\frac{1}{2}(b-a)+\left|x_{1}-\frac{a+b}{2}\right|\right] V_{a}^{b}(f)
$$

for all $x_{1} \in[a, b]$.

We note that the best inequality we can get in (4.6) is for $x_{1}=(a+b) / 2$ obtaining the "midpoint inequality"

$$
\left|\int_{a}^{b} f(x) d x-f\left(\frac{a+b}{2}\right)(b-a)\right| \leqslant \frac{1}{2}(b-a) V_{a}^{b}(f)
$$

(b) If we choose in (4.5) $\alpha_{1}=(5 a+b) / 6, \alpha_{2}=(a+5 b) / 6$ and $x_{1} \in[(5 a+b) / 6$, $(a+5 b) / 6]$, then we get

$$
\begin{array}{r}
\mid \begin{array}{r}
\mid \int_{a}^{b} f(x) d x-\frac{b-a}{3}\left[\frac{f(a)+f(b)}{2}\right. \\
\left.+2 f\left(x_{1}\right)\right] \mid
\end{array} \\
+\max \left\{\left|x_{1}-\frac{2 a+b}{2}\right|,\left|\frac{1}{2} \cdot(b-a)+\right| x_{1}-\frac{a+b}{2} \mid\right. \\
\end{array}
$$

Particularly, if we choose in (4.8), $x_{1}=(a+b) / 2$, then we get the following "Simpson's rule inequality"

$$
\left|\int_{a}^{b} f(x) d x-\frac{b-a}{3}\left[\frac{f(a)+f(b)}{2}+2 f\left(\frac{a+b}{2}\right)\right]\right| \leqslant \frac{1}{3}(b-a) V_{a}^{b}(f) .
$$




\section{SOME COMPOSITE QUADRATURE FORMULAE}

Let us consider the partition of the interval $[a, b]$ given by $\Delta_{n}: a=x_{0}<$ $x_{1}<\ldots<x_{n-1}<x_{n}=b$ and put $h_{i}:=x_{i+1}-x_{i}(i=0, \ldots, n-1)$ and $\nu(h):=$ $\max \left\{h_{i} \mid i=0, \ldots, n-1\right\}$.

The following theorem holds.

THEOREM 5. Let $f:[a, b] \rightarrow \mathbf{R}$ be of bounded variation on $[a, b]$ and $k \geqslant 1$. Then we have the composite quadrature formula

$$
\int_{a}^{b} f(x) d x=A_{k}\left(\Delta_{n}, f\right)+R_{k}\left(\Delta_{n}, f\right)
$$

where

$$
A_{k}\left(\Delta_{n}, f\right):=\frac{1}{k}\left[T\left(\Delta_{n}, f\right)+\sum_{i=0}^{n} \sum_{j=1}^{k-1} f\left[\frac{(k-j) x_{i}+j x_{i+1}}{k}\right] h_{i}\right]
$$

and

$$
T\left(\Delta_{n}, f\right):=\frac{1}{2} \sum_{i=0}^{n-1}\left[f\left(x_{i}\right)+f\left(x_{i+1}\right)\right] h_{i}
$$

is the trapezoid quadrature formula.

The remainder $R_{k}\left(\Delta_{n}, f\right)$ satisfies the estimate

$$
\left|R_{k}\left(\Delta_{n}, f\right)\right| \leqslant \frac{1}{2 k} \nu(h) V_{a}^{b}(f) .
$$

Proof: Applying Corollary 2 on the intervals $\left[x_{i}, x_{i+1}\right](i=0, \ldots, n-1)$ we get

$$
\begin{aligned}
\left|\int_{x_{i}}^{x_{i+1}} f(x) d x-\left[\frac{1}{k} \frac{f\left(x_{i}\right)+f\left(x_{i+1}\right)}{2} h_{i}+\frac{h_{i}}{k} \sum_{j=1}^{k} f\left[\frac{(k-j) x_{i}+j x_{i+1}}{k}\right]\right]\right| & \\
& \leqslant \frac{1}{2 k} h_{i} V_{x_{i}}^{x_{i+1}}(f) .
\end{aligned}
$$

Now, using the generalised triangle inequality, we get:

$$
\begin{aligned}
& \left|R_{k}\left(\Delta_{n}, f\right)\right| \\
& \quad \leqslant \sum_{i=0}^{n-1}\left|\int_{x_{i}}^{x_{i+1}} f(x) d x-\left[\frac{1}{k} \cdot \frac{f\left(x_{i}\right)+f\left(x_{i+1}\right)}{2} h_{i}+\frac{h_{i}}{k} \sum_{j=1}^{k-1} f\left[\frac{(k-j) x_{i}+j x_{i+1}}{k}\right]\right]\right| \\
& \quad \leqslant \frac{1}{2 k} \sum_{i=0}^{n-1} h_{i} V_{x_{i}}^{x_{i+1}}(f) \leqslant \frac{\nu(h)}{2 k} \sum_{i=0}^{n-1} V_{x_{i}}^{x_{i+1}}(f)=\frac{\nu(h)}{2 k} V_{a}^{b}(f)
\end{aligned}
$$

and the theorem is proved.

The following corollaries hold. 
COROLLARY 4. Let $f$ be as above. Then we have the formula:

$$
\int_{a}^{b} f(x) d x=\frac{1}{2}\left[T_{n}\left(\Delta_{n}, f\right)+M_{n}\left(\Delta_{n}, f\right)\right]+R_{2}\left(\Delta_{n}, f\right)
$$

where $M_{n}\left(\Delta_{n}, f\right)$ is the midpoint quadrature formula,

$$
M_{n}\left(\Delta_{n}, f\right):=\sum_{i=0}^{n-1} f\left(\frac{x_{i}+x_{i+1}}{2}\right) h_{i}
$$

and the remainder $R_{2}\left(\Delta_{n}, f\right)$ satisfies the inequality:

$$
\left|R_{2}\left(\Delta_{n}, f\right)\right| \leqslant \frac{1}{4} \nu(h) V_{a}^{b}(f)
$$

COROLlary 5. Under the above assumptions we have

$$
\begin{aligned}
\int_{a}^{b} f(x) d x=\frac{1}{3} & {\left[T_{n}\left(\Delta_{n}, f\right)+\sum_{i=0}^{n-1} f\left(\frac{2 x_{i}+x_{i+1}}{3}\right) h_{i}+\sum_{i=0}^{n-1} f\left(\frac{x_{i}+2 x_{i+1}}{3}\right) h_{i}\right] } \\
& +R_{3}\left(\Delta_{n}, f\right) .
\end{aligned}
$$

The remainder $R_{3}\left(\Delta_{n}, f\right)$ satisfies:

$$
\left|R_{3}\left(\Delta_{n}, f\right)\right| \leqslant \frac{1}{6} \nu(h) V_{a}^{b}(f)
$$

The following theorem holds:

THEOREM 6. Let $f$ and $\Delta_{n}$ be as above and $\xi_{i} \in\left[x_{i}, x_{i+1}\right](i=0, \ldots, n-1)$. Then we have the quadrature formula:

$$
\int_{a}^{b} f(x) d x=\sum_{i=0}^{n-1}\left[\left(\xi_{i}-x_{i}\right) f\left(x_{i}\right)+\left(x_{i+1}-\xi_{i}\right) f\left(x_{i+1}\right)\right]+R\left(\xi, \Delta_{n}, f\right) .
$$

The remainder $R\left(\xi, \Delta_{n}, f\right)$ satisfies:

$$
\begin{aligned}
\left|R\left(\xi, \Delta_{n}, f\right)\right| & \leqslant\left[\frac{1}{2} \nu(h)+\max \left\{\left|\xi_{i}-\frac{x_{i}+x_{i+1}}{2}\right|, i=0, \ldots, n-1\right\}\right] V_{a}^{b}(f) \\
& \leqslant \nu(h) V_{a}^{b}(f) .
\end{aligned}
$$

for all $\xi_{i}$ as above.

Proof: Apply Proposition 1 on the interval $\left[x_{i}, x_{i+1}\right](i=0, \ldots, n-1)$ to get

$$
\begin{aligned}
\left|\int_{x_{i}}^{x_{i+1}} f(x) d x-\left[\left(\xi_{i}-x_{i}\right) f\left(x_{i}\right)+\left(x_{i+1}-\xi_{i}\right) f\left(x_{i+1}\right)\right]\right| \\
\leqslant\left[\frac{1}{2} h_{i}+\max \left\{\left|\xi_{i}-\frac{x_{i}+x_{i+1}}{2}\right|\right\}\right] V_{x_{i}}^{x_{i+1}}(f) .
\end{aligned}
$$


Summing over $i$ from 0 to $n-1$, using the generalised triangle inequality and the properties of the maximum mapping, we get (5.10).

Corollary 6. Let $f$ and $\Delta_{n}$ be as above. Then we have

(1) the "left rectangle rule"

$$
\int_{a}^{b} f(x) d x=\sum_{i=0}^{n-1} f\left(x_{i}\right) h_{i}+R_{l}\left(\Delta_{n}, f\right) ;
$$

(2) the "right rectangle rule"

$$
\int_{a}^{b} f(x) d x=\sum_{i=0}^{n-1} f\left(x_{i+1}\right) h_{i}+R_{r}\left(\Delta_{n}, f\right)
$$

(3) the "trapezoid rule"

$$
\int_{a}^{b} f(x) d x=T\left(\Delta_{n}, f\right)+R_{T}\left(\Delta_{n}, f\right)
$$

where

$$
\left|R_{l}\left(\Delta_{n}, f\right)\right|\left|R_{r}\left(\Delta_{n}, f\right)\right| \leqslant \nu(h) V_{a}^{b}(f)
$$

and

$$
\left|R_{T}\left(\Delta_{n}, f\right)\right| \leqslant \frac{1}{2} \nu(h) V_{a}^{b}(f)
$$

The following theorem also holds.

THEOREM 7 . Let $f$ and $\Delta_{n}$ be as above and $\xi_{i} \in\left[x_{i}, x_{i+1}\right], x_{i} \leqslant \alpha_{i}^{(1)} \leqslant \xi_{i}$ $\leqslant \alpha_{i}^{(2)} \leqslant x_{i+1}$. Then we have the quadrature formula:

$$
\begin{aligned}
\int_{a}^{b} f(x) d x= & \sum_{i=0}^{n-1}\left(\alpha_{i}^{(1)}-x_{i}\right) f\left(x_{i}\right)+\sum_{i=0}^{n-1}\left(\alpha_{i}^{(2)}-\alpha_{i}^{(1)}\right) f\left(\xi_{i}\right) \\
& +\sum_{i=0}^{n-1}\left(x_{i+1}-\alpha_{i}^{(2)}\right) f\left(x_{i+1}\right)+R\left(\xi, \alpha_{i}^{(1)}, \alpha_{i}^{(2)}, \Delta_{n}, f\right) .
\end{aligned}
$$

The remainder $R\left(\xi, \alpha_{i}^{(1)}, \alpha_{i}^{(2)}, \Delta_{n}, f\right)$ satisfies

$$
\begin{aligned}
\mid R(\xi, & \left.\alpha_{i}^{(1)}, \alpha_{i}^{(2)}, \Delta_{n}, f\right) \mid \\
\leqslant & \left\{\frac{1}{2}\left[\frac{1}{2} \nu(h)+\max _{i=0, \ldots, n-1}\left|\xi_{i}-\frac{x_{i}+x_{i+1}}{2}\right|\right]\right. \\
& \left.+\max \left\{\max _{i=0, \ldots, n-1}\left|\alpha_{i}^{(1)}-\frac{x_{i}+\xi_{i}}{2}\right|, \max _{i=0, \ldots, n-1}\left|\alpha_{i}^{(2)}-\frac{\xi_{i}+x_{i+1}}{2}\right|\right\}\right\} V_{a}^{b}(f) \\
\leqslant & {\left[\frac{1}{2} \nu(h)+\max _{i=0, \ldots n-1}\left|\xi_{i}-\frac{x_{i}+x_{i+1}}{2}\right|\right]\left\|f^{\prime}\right\|_{1} \leqslant \nu(h) V_{a}^{b}(f) . }
\end{aligned}
$$


Proof: Apply Proposition 2 on the interval $\left[x_{i}, x_{i+1}\right]$ to obtain

$$
\begin{aligned}
& \left|\int_{x_{i}}^{x_{i+1}} f(x) d x-\left[\left(\alpha_{i}^{(1)}-x_{i}\right) f\left(x_{i}\right)+\left(\alpha_{i}^{(2)}-\alpha_{i}^{(1)}\right) f\left(\xi_{i}\right)+\left(x_{i+1}-\xi_{i}\right) f\left(x_{i+1}\right)\right]\right| \\
& \leqslant \frac{1}{2}\left[\frac{1}{2} h_{i}+\left|\xi_{i}-\frac{x_{i}+x_{i+1}}{2}\right|+\max \left\{\left|\alpha_{i}^{(1)}-\frac{x_{i}+\xi_{i}}{2}\right|,\left|\alpha_{i}^{(2)}-\frac{\xi_{i}+x_{i+1}}{2}\right|\right\}\right] V_{x_{i}}^{x_{i+1}}(f) .
\end{aligned}
$$

Summing over $i$ from 0 to $n-1$ and using the properties of modulus and maximum we get the desired inequality.

We shall omit the details.

The following corollary is the result of Dragomir from the recent paper [2].

COROLlaRY 7. Under the above assumptions, we have the Riemann's quadrature formula:

$$
\int_{a}^{b} f(x) d x=\sum_{i=0}^{n-1} f\left(\xi_{i}\right) h_{i}+R_{R}\left(\xi, \Delta_{n}, f\right) .
$$

The remainder $R_{R}\left(\xi, \Delta_{n}, f\right)$ satisfies

$$
\begin{aligned}
\left|R_{R}\left(\xi, \Delta_{n}, f\right)\right| & \leqslant\left[\frac{1}{2} \nu(h)+\max \left\{\left|\xi_{i}-\frac{x_{i}+x_{i+1}}{2}\right|, i=0, \ldots, n-1\right\}\right] V_{a}^{b}(f) \\
& \leqslant \nu(h) V_{a}^{b}(f) .
\end{aligned}
$$

for all $\xi_{i} \in\left[x_{i}, x_{i+1}\right](i=0, \ldots, n)$.

Finally, the following corollary which generalises Simpson's quadrature formula holds.

Corollary 8. Under the above assumptions and if $\xi_{i} \in\left[\left(x_{i+1}+5 x_{i}\right) / 6\right.$, $\left.\left(x_{i}+5 x_{i+1}\right) / 6\right](i=0, \ldots, n-1)$, then we have the formula:

$$
\int_{a}^{b} f(x) d x=\frac{1}{6} \sum_{i=0}^{n-1}\left[f\left(x_{i}\right)+f\left(x_{i+1}\right)\right] h_{i}+\frac{2}{3} \sum_{i=0}^{n-1} f\left(\xi_{i}\right) h_{i}+S\left(f, \Delta_{n}, \xi\right) .
$$

The remainder $S\left(f, \Delta_{n}, \xi\right)$ satisfies

(5.19) $\left|S\left(f, \Delta_{n}, \xi\right)\right|$

$$
\begin{aligned}
\leqslant & \left\{\frac { 1 } { 2 } \left[\frac{\nu(h)}{2}+\max _{i=0, \ldots, n-1}\left\{\left|\xi_{i}-\frac{x_{i}+x_{i+1}}{2}\right|\right\}\right.\right. \\
& \left.\left.+\max \left\{\max _{i=0, \ldots, n-1}\left|\xi_{i}-\frac{2 x_{i}+x_{i+1}}{3}\right|, \max _{i=0, \ldots, n-1}\left|\frac{x_{i}+2 x_{i+1}}{3}-\xi_{i}\right|\right\}\right]\right\} V_{a}^{b}(f) .
\end{aligned}
$$

The proof follows by the inequality (4.8) and we omit the details. 
REMARK 3. Now, if we choose in (5.18), $\xi_{i}=\left(x_{i}+x_{i+1}\right) / 2$, then we get "Simpson's quadrature formula"

$$
\int_{a}^{b} f(x) d x=\frac{1}{6} \sum_{i=0}^{n-1}\left[f\left(x_{i}\right)+f\left(x_{i+1}\right)\right] h_{i}+\frac{2}{3} \sum_{i=0}^{n-1} f\left(\frac{x_{i}+x_{i+1}}{2}\right) h_{i}+S\left(f, \Delta_{n}\right)
$$

where the remainder term $S\left(f, \Delta_{n}\right)$ satisfies

$$
\left|S\left(f, \Delta_{n}\right)\right| \leqslant \frac{1}{3} \nu(h) V_{a}^{b}(f) .
$$

\section{REFERENCES}

[1] S.S. Dragomir, 'A generalization of Ostrowski integral inequality for mappings whose derivatives belong to $L_{\infty}[a, b]$ and applications in numerical integration', (submitted).

[2] S.S. Dragomir, 'A generalization of Ostrowski integral inequality for mappings of bounded variation', (submitted).

[3] S.S. Dragomir and S. Wang, 'A new inequality of Ostrowski's type in $L_{1}-$ norm and applications to some special means and to some numerical quadrature rules', Tamkang J. Math. 28 (1997), 239-244.

[4] S.S. Dragomir and S. Wang, 'An inequality of Ostrowski-Grüss' type and its applications to the estimation of error bounds for some special means and for some numerical quadrature rules', Computers Math. Appl. 33 (1997), 15-20.

[5] S.S. Dragomir and S. Wang, 'Applications of Ostrowski's inequality to the estimation o error bounds for some special means and some numerical quadrature rules', Appl. Math. Lett. 11 (1998), 105-109.

[6] S.S. Dragomir and S. Wang, 'A new inequality of Ostrowski's type in $L_{p}$-norm and applications to some special means and to some numerical quadrature rules', Indian $J$. Math. (to appear).

[7] D.S. Mitrinović, J.E. Pečarić and A.M. Fink, 'Inequalities involving functions and their integrals and derivatives' (Kluwer Academic Publishers, Dordrecht).

School of Communication and Informatics

Victoria University of Technology

PO Box 14428

MCMC Melbourne City, Vic 3001

Australia

e-mail: sever@matilda.vu.edu.au

http://matilda.vut.edu.au/ ${ }^{-}$rgmia/dragomirweb.html 